\title{
Mortal neutropenic enterocolitis in acute lymphoblastic leukemia
}

\author{
Omer Elcioglu, Abdullah Ozkok, Timur Akpinar, Reyhan Kucukkaya \\ Division of Hematology, Department of Internal Medicine, Istanbul Faculty of Medicine, Istanbul University, Istanbul, Turkey \\ Email: o.c.elcioglu@gmail.com, abdullahozkok@yahoo.com, doktortimur@gmail.com, rkucukkaya@hotmail.com
}

Received 30 March 2013; revised 30 April 2013; accepted 7 May 2013

Copyright (C) 2013 Omer Elcioglu et al. This is an open access article distributed under the Creative Commons Attribution License, which permits unrestricted use, distribution, and reproduction in any medium, provided the original work is properly cited.

\begin{abstract}
Neutropenic enterocolitis (NE) or typhlitis is a serious complication of anticancer therapy, especially in hematological malignancies. We describe a case of acute lymphoid leukemia presenting the early onset of a syndrome with the clinical features and specific radiological findings of $\mathrm{NE}$ during the neutropenic phase.
\end{abstract}

Keywords: Acute Lymphoblastic Leukemia; Chemotherapy; Neutropenic Enterocolitis

\section{INTRODUCTION}

Neutropenic enterocolitis (NE) was first described in 1970s as a complication of childhood leukemia treatment [1]. It is a life-threatening complication of chemotherapy, which can lead to death in immunocompromised patients. The frequency and mortality of NE in patients with acute leukemia are $2.35 \%-6.5 \%$ and $22.3 \%-50 \%$ respectively [2]. The diagnosis of NE may be delayed because the presenting clinical features (fever, abdominal pain and diarrhea) are not specific and may suggest other abdominal diseases. To support the clinical diagnosis of NE, imaging techniques including abdominal ultrasonography and computed tomography (CT) are used [3]. Herein we present a case of a patient with acute lymphoid leukemia (ALL) presenting the early onset of a syndrome with the clinical features and specific radiological findings of $\mathrm{NE}$, during the neutropenic phase.

\section{CASE REPORT}

A 17-year-old female was admitted with relapsed T-cell acute lymphoblastic leukemia (T-ALL). Earlier, she was treated wtih with daunomycin, vincristine, methylprednisolone, mercaptopurine and intrathecal methotrexate and remisson was obtained. On physical examination, the patient appeared toxic. No cervical, axillary, or inguinal lymph node enlargement was noted. The only remarkable finding on examination was hepatosplenomegaly. Laboratory analysis revealed a white blood cell count of $415,800 / \mu \mathrm{L}$, with a neutrophil count of $101,100 / \mu \mathrm{L}$ and a lymphocyte count of $268,200 / \mu \mathrm{L}$, a platelet count of $50,000 / \mu \mathrm{L}$, a serum hemoglobin level of $10.2 \mathrm{~g} / \mathrm{dL}$. Peripheral blood smears showed evidence of lymphoblastic leukemia. Erythrocyte sedimentation rate (ESR) of 24 $\mathrm{mm} / \mathrm{hr}$, C-reactive protein $50 \mathrm{mg} / \mathrm{L}$, aspartate aminotransferase (AST), $1272 \mathrm{IU} / \mathrm{L}$ (normal range 3 - $45 \mathrm{IU} / \mathrm{L}$ ); alanine aminotransferase (ALT), $442 \mathrm{IU} / \mathrm{L}$ (normal range 5 - $35 \mathrm{IU} / \mathrm{L}$ ), alkaline phosphatase level at $309 \mathrm{IU} / \mathrm{L}$ (range, 20 - $140 \mathrm{IU} / \mathrm{L}$ ), lactate dehydrogenase (LDH) of $12,000 \mathrm{Units} / \mathrm{L}$ (range, $80-260 \mathrm{IU} / \mathrm{L}$ ). The rest of the biochemical tests were unremarkable. Induction therapy with high dose fludarabine, cytarabine and dexamethasone were started. Neutropenia developed in the second week of the therapy. During the neutropenic phase (neutrophil counts of $100 / \mu \mathrm{L}$ ), patient had fever higher than $38^{\circ} \mathrm{C}$. Parenteral cefepime hydrochloride and amicacin were started with febrile neutropenia diagnosis. Diarrhea started during follow-up. Stool microscopy did not reveal any evidence of infection and stool cultures remained sterile. Symptomatic treatment for diarrhea was given. Then, acute severe abdominal pain developed. Physical examination showed decreased bowel sounds with widespread abdominal tenderness. Marked intestinal distention was observed in direct abdominal X-ray (Figure 1A). Abdominal computed tomography revealed increased intestinal wall thickness of $10 \mathrm{~mm}$ (Figure 1B). During this time neutrophil count was $100 \mu \mathrm{L}$, and serum, urine amylase and serum lipase levels were normal. The diagnosis of NE was made and total parenteral nutrition (TPN) was started with cessation of oral intake. Imipenem, teicoplanin, metronidazole, amikacin, amphotericin $\mathrm{B}$ together with granulocyte colony stimulating factor (G-CSF) were also administered. She was followed in intensive care unit. Surgical intervention was not considered because the overall situation was not good. How- 


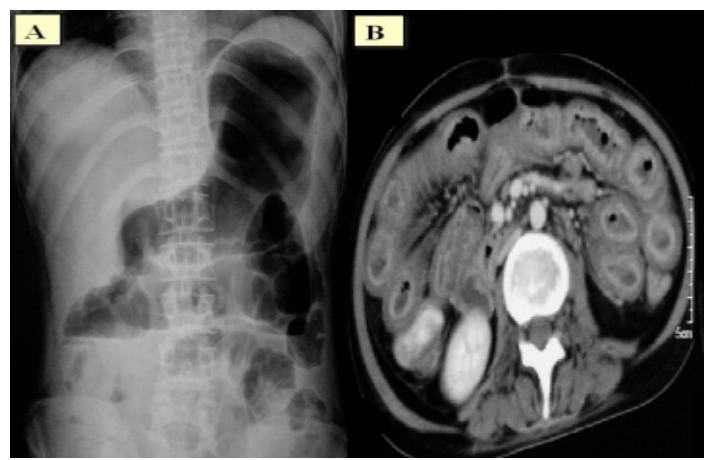

Figure 1. Direct abdominal X-ray showed marked intestinal distention (A), and abdominal CT revealed increased intestinal wall thickness of $10 \mathrm{~mm}(\mathrm{~B})$.

ever five days later, she died due to septic shock.

\section{DISCUSSION}

$\mathrm{NE}$ is a frequent complication of intensive chemotherapy in acute leukemia. There is no consensus on diagnostic criteria. Suggested diagnosic criteria are a combination of clinical and radiological criteria to define illness including fever, abdominal pain and any bowel wall thickening $>4 \mathrm{~mm}$ detected by ultrasonography or computed tomography [4]. Our patient had these three elements. The mortality rate is high, especially in patients with mural thickness of more than $10 \mathrm{~mm}$ [5]. Conservative management should consist of bowel rest, TPN, broad-spectrum antibiotherapy and G-CSF [6]. Systemic antifungal agents should be given empirically in neutropenic enterocolitis. Antifungal agents appear to be beneficial for reducing mortality, need for surgical intervention and diarrhea [7].

The pathogenesis of NE is probably complex which include neutropenia (the main contributing factor), anemia-resulting with ischemia of bowel wall, leukemic infiltrations in bowel wall, as well as rapid regression of leukemic infiltrations induced by potent treatment which may cause localized necrosis and facilitate bacterial translocation. The major predisposing factors are prolonged neutropenia and cytotoxic agent-induced mucosal epithelial cell necrosis. The other factors are intramural hemorrhage due to severe thrombocytopenia, a shift in the normal gastrointestinal microbial flora due to antibiotics and antifungal drugs, nosocomial colonization by hospital flora, and paralytic ileus caused by some drugs e.g., vincristine and cytosine arabinoside. The combined loss of neutrophils and epithelial integrity make the bowel very susceptible to bacterial invasion, infarction, and occasionally perforation. Mucosal neoplastic infiltrates and ischaemia also play a role in the genesis of the lesion [6]. Although surgical treatment is generally not recommended, in some cases, it may be required: 1) persistent gastrointestinal bleeding in spite of the resolution of neutropenia, thrombocytopenia, or clotting abnormalities; 2) free intraperitoneal perforation; and 3) clinical deterioration suggesting uncontrolled sepsis $[6,8]$. The standard surgical approach is a two-stage right hemicolectomy [6].

The disorder is often fatal and mortality rates can reach up to $50 \%$. Therefore, early diagnosis and treatment are very important. There is no consensus about the treatment therefore care of these patients should be individualized. Most patients can be managed conservatively with bowel rest, total parenteral nutrition, intravenous antibiotics, and recombinant granulocyte colony-stimulating factor. Surgical treatment is only recommended for the aforementioned cases.

\section{REFERENCES}

[1] Wagner, M.L., Rosenberg, H.S., Fernbach, D.J. and Singleton, E.B. (1970) Typhlitis a complication of leukemia in childhood. American Journal of Roentgenology, 109, 341-350.

doi:10.2214/ajr.109.2.341

[2] Gorschluter, M., Glasmacher, A., Hahn, C., Leutner, C., Marklein, G., Remig, J., Schmidt-Wolf, I.G. and Sauerbruch, T. (2001) Severe abdominal infection in neutropenic patients. Cancer Investigation, 19, 669-677. doi:10.1081/CNV-100106141

[3] Tjon, A., Tham, R.T., Vlasveld, L.T. and Willemze, R. (1990) Gastrointestinal complications of cytosine-arabinoside chemotherapy: Findings on plain abdominal radiographs. American Journal of Roentgenology, 154, 9598. doi:10.2214/ajr.154.1.2104733

[4] Gorschluter, M., Mey, U., Strehl, J., Ziske, C., Schepke, M., Schmidt-Wolf, I.G., Sauerbruch, T. and Glasmacher, A. (2005) Neutropenic enterocolitis in adults: Systematic analysis of evidence quality. European Journal of Haematology, 75, 1-13. doi:10.1111/j.1600-0609.2005.00442.x

[5] Cartoni, C., Dragoni, F., Micozzi, A., Pescarmona, E., Mecarocci, S., Chiletti, P., Petti, M.C., Meloni, G. and Mandelli, F. (2001) Neutropenic enterocolitis in patients with acute leukemia: Prognostic significance of bowel wall thickening detected by ultrasonography. Journal of Clinical Oncology, 19, 756-761.

[6] Davila, M.L. (2006) Neutropenic enterocolitis. Current Opinion in Gastroenterology, 22, 44-47.

[7] Cardona, A.F., Ramos, P.L. and Casasbuenas, A. (2005) From case reports to systematic reviews in neutropenic enterocolitis. European Journal of Haematology, 75, 445446.

doi:10.1111/j.1600-0609.2005.00544.x

[8] Gomez, L., Martino, R. and Rolston, K.V. (1998) Neutropenic enterocolitis: Spectrum of the disease and comparison of definite and possible cases. Journal of Clinical Investigation, 27, 695-699. 